\title{
Turning lead into gold: reprogramming retinal cells to cure blindness
}

\author{
Seth Blackshaw ${ }^{1,2,3,4,5}$ and Joshua R. Sanes ${ }^{6,7}$ \\ ${ }^{1}$ Departments of Neuroscience, ${ }^{2}$ Neurology, and ${ }^{3}$ Ophthalmology, ${ }^{4}$ Institute for Cell Engineering, and ${ }^{5}$ Kavli Neuroscience Discovery Institute, Johns Hopkins University School of Medicine, Baltimore, \\ Maryland, USA. 'enter for Brain Science and ’Department of Molecular and Cellular Biology, Harvard University, Cambridge, Massachusetts, USA.
}

$\mathbf{T}_{\mathrm{t}}$ he vast majority of irreversible vision loss results from the death of cells in the retina, an outpost of the central nervous system at the back of the eye. Two of the three most prevalent retinal diseases, age-related macular degeneration and diabetic retinopathy, lead to loss of light-sensing rod and cone photoreceptors; the third, glaucoma, leads to loss of the retinal ganglion cells (RGCs) that carry visual information from the eye to the brain. These three diseases together afflict nearly 15 million people in the United States and their prevalence is increasing as the population ages. Many more are affected by monogenic photoreceptor dystrophies, each rare but substantial in aggregate. Treatments that slow progression are available for some of these diseases, but since there are currently no available therapies that can restore visual function following the loss of retinal neurons, the prospect of generating new neurons to treat a variety of degenerative retinal diseases has elicited enormous excitement.

\section{Preclinical models of cell-based therapies}

Most efforts at so-called cell therapies involve transplantation of cells derived from stem cells or other sources. This approach is conceptually straightforward, but has proven technically challenging and led to false starts (1). Most notably, several high-profile studies which reported that transplanted immature photoreceptor precursors functionally integrated into the host retina were later shown to actually reflect cytoplasmic transfer from donor to host cells, with little or no integration of donor photoreceptors occurring (Figure 1). An alternative and complementary approach is to replace lost cells by inducing proliferation of endogenous cells and then reprogramming them to generate neurons that have been lost. Hope for this approach arose from studies of lower vertebrates, in which Müller glia (MG), the endogenous retinal glial type, can be efficiently reprogrammed into neurogenic progenitors, which then differentiate in situ to form multiple retinal neuronal classes, including photoreceptors, interneurons (bipolar and amacrine cells) that process visual information, and RGCs (2).

Over the past few years, a flurry of studies has built on these findings, manipulating key regulators of gene expression to reprogram adult rodent MG (3-9), as well as amacrine cells (10), resulting in generation of functional retinal neurons. The experimental paradigm is similar in all cases: researchers use adeno-associated viruses (AAVs) as vectors with cell type-specific promoters (7-9) or Cre-expressing transgenic lines $(3-5,10)$ to selectively target the cells to be reprogrammed. They then exploit this genetic access to introduce fluorescent reporters that track the cells and their progeny, along with interventions (overexpressed cDNAs, shRNAs, gRNAs, or conditional knockouts) to induce reprogramming (Figure 1). The reporter is then used to target reprogrammed cells for structural, functional, and molecular analysis.

Despite this methodological similarity, however, the results differ greatly. Three groups report generation of RGCs from MG $(7,8)$ or interneurons (10) by depletion of Ptbp1 (7) or ectopic expression of Pou4f2 (Brn3b) plus Atoh7 (Math5) (8) or Pou $4 f 2$ plus Sox $4(8,10)$. Two groups generated bipolar and amacrine-like cells by

Conflict of interest: SB is a cofounder and stockholder of CDI Labs. JRS is a consultant for Biogen.

overexpression of $\operatorname{Ascl1}(3,4)$ or conditional deletion of three nuclear factor I (NFI) class transcription factors (5). Another two groups generated photoreceptors - rods by priming with $\beta$-catenin followed by ectopic expression of Otx, Crx , and $\mathrm{Nrl}(9)$ and cones by deletion of Ptbp1 (6).

\section{Interpreting divergent results}

These results are exciting, but could they all be correct? Inconsistencies among them, along with previous work in the field, suggest that the answer is no. First, AAV-mediated knockdown or deletion of Ptbp1, which encodes an RNA-binding protein expressed by MG, led to selective generation of cone photoreceptors in one study and RGCs in another $(6,7)$. Second, the newly generated neurons were reported to be morphologically, functionally, and molecularly indistinguishable from wild-type neurons in some studies (6-10) but abnormal and immature in others $(3-5)$. Third, three studies reported that newly generated RGCs rapidly extended axons through the optic nerve to synapse on central targets and mediate visually evoked behaviors $(7,8,10)$, despite the fact that regeneration of axotomized RGCs is highly inefficient in adult mammalian retina and the very limited number of regenerating axons seldom reach central targets (11).

Sorting out these claims is important to understanding the underlying mechanisms and improving protocols. It is also critically important from a clinical perspective; as noted above, death of RGCs and photoreceptors accounts for the vast majority of irreversible vision loss. In contrast, few blinding diseases, and no prevalent ones, result from loss of interneurons. Put simply, the ability to generate photoreceptors or RGCs from endogenous MG would be far more useful than the ability to generate amacrine and bipolar cells. 
A Material transfer vs. integration

\section{Observation}

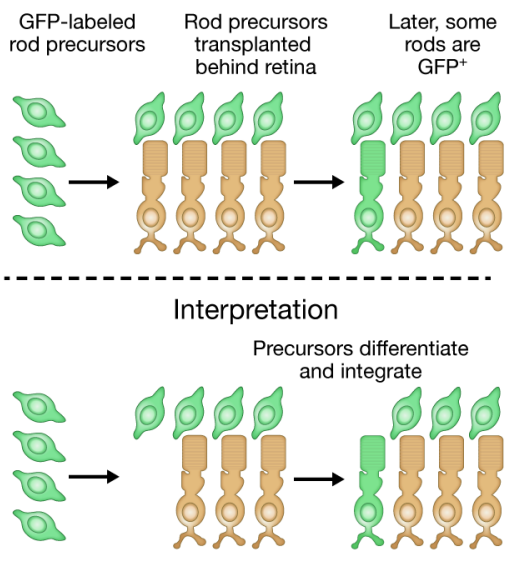

Alternative interpretation

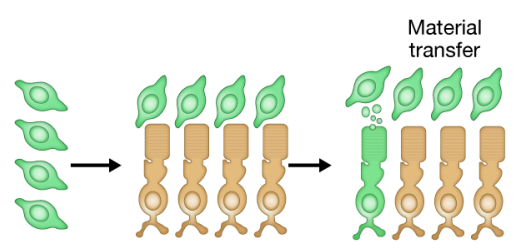

B Cell targeting vs. cell generation

\section{Observation}

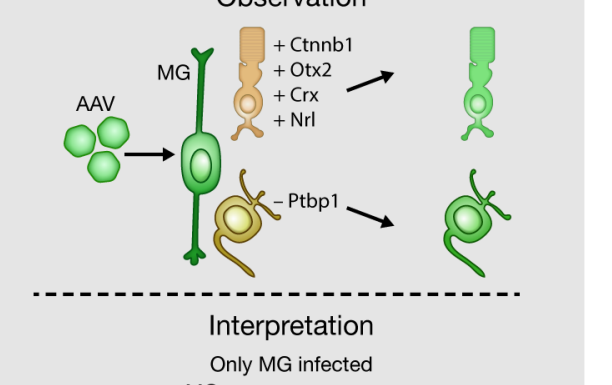

MG generates neurons

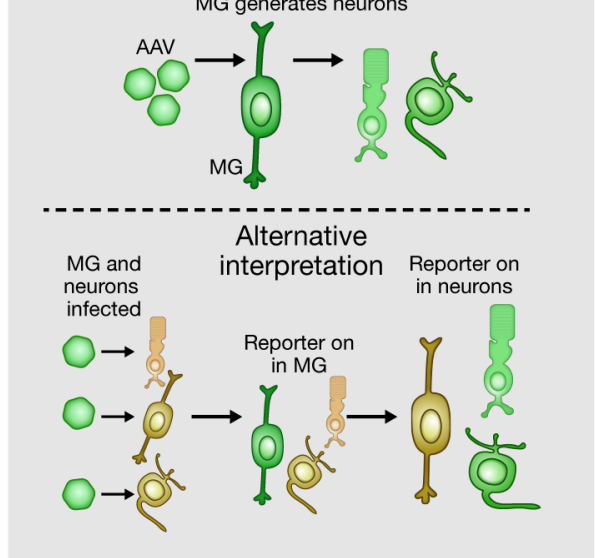

C Trophic support vs. replacement
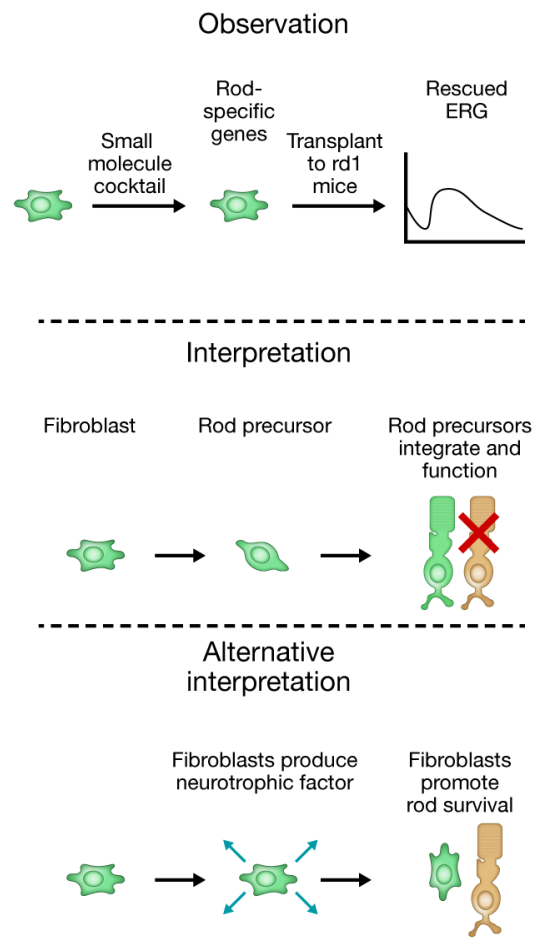

Figure 1. Summary of three different mechanisms by which reported observations of photoreceptor and RGC replacement are known or proposed to reflect alternative effects. Observation of $\mathrm{GFP}^{+}$photoreceptors in host retinas following transplantation of GFP+ donor photoreceptor precursors was initially interpreted as evidence of integration of transplanted photoreceptors, but was later shown to actually reflect cytoplasmic transfer from donor to host. More recent studies reporting AAV-mediated reprogramming of Müller glia to photoreceptors or RGCs may instead reflect ectopic expression of glia-specific minipromoter constructs in retinal neurons. Finally, studies reporting rescue of photoreceptor function in rd1 mice following transplantation of fibroblasts treated with small molecules to induce expression of photoreceptor-specific genes may reflect neurotrophic effects of transplanted cells. ERG, electroretinogram.

What accounts for these strikingly different outcomes? One likely source of these discrepancies is the nature of the reagents used. The AAVs employed in these studies (6-9) transduce both neurons and glia, with restriction of expression to MG dependent on use of appropriate glia-specific promoters. Conclusions drawn about lineage relationships between glia and neurons thus depend on the absolute cell specificity of the promoters. It is clear that the specificity of expression can be influenced by the adjacent cDNA sequence, the viral concentration, and the time after infection (12). All reports assessed specificity to some extent, but expression in a small fraction of endogenous photoreceptors or RGCs could account for the results. Because these studies did not test whether reprogrammed cells actually express molecular markers of retinal progenitors or immature neurons during the process of converting from MG to neurons, it will be important that they be supplemented with rigorous, genetic-based cell lineage analysis and single-cell RNA sequencing (scRNA-Seq) analysis to provide convincing evidence of specificity. The danger is a real one, as highlighted in a recent study that reexamined claims of direct reprogramming of astrocytes into neurons by Neurod1-mediated AAV transduction; it concluded that results actually reflected a spurious lineage relationship resulting from progressive silencing of glia-specific minipromoter-regulated reporter constructs in astrocytes followed by their later ectopic expression in neurons (13). Ensuring that the reprogrammed cells were indeed generated after treatment, using BrdU or EdU labeling, would be useful in this regard. Independent replication of these results is also critical for evaluating their accuracy and potential usefulness in preclinical studies.
Cre-expressing transgenic lines used to restrict expression in other studies (3-5, 10) are subject to similar caveats regarding specificity of expression. In these cases, however, the cell-type specificity of the transgenes had been rigorously established in previous studies, and scRNASeq analysis was used to demonstrate the presence of bipolar and amacrine-like precursors. At present then, we conclude that there is more solid support for reprogramming of MG into interneurons than into RGCs or photoreceptors.

Related concerns apply to another recent study, which puts a new twist on using cell transplantation to treat photoreceptor dystrophies (14). In this study, a drug cocktail was used to induce fibroblasts to express a subset of photoreceptor-specific genes in vitro (Figure 1). Transplantation of these treated cells improved photoreceptor function in mice with photoreceptor dystrophy. However, these cells did not 
show classical photoreceptor morphology or clearly integrate into retinal circuitry. They were not characterized by scRNASeq following transplantation and effects of transplanting untreated fibroblasts were not tested. This leaves open the possibility that transplanted cells might have reduced photoreceptor death and/or improved function of native cells by releasing a neuroprotective factor (Figure 1). This is another study that could have benefited from a more developmental approach, such as analyzing whether changes in cell death contribute to observed changes in cell numbers. Unambiguous determination of how transplanted cells improve visual function is a difficult task. One possibility would be to include an engineered "kill switch," such as an inhibitory designer receptor exclusively activated by designer drugs (DREADD) (15) in the donor cells, to acutely and selectively block their electrical activity so that circuit-based and trophic contributions could be distinguished.

\section{Conclusions}

Studies aimed at replacing retinal neurons - whether they involve transplanted neural precursors or reprogrammed glia - attempt to replicate the processes that normally occur as the retina develops. It is therefore important to take advantage of what we have learned from decades of developmental studies in interpreting the new results. It is possible that interventions will provide benefit by unanticipated mechanisms, but we need to know if or when this is the case. In studies of photoreceptor transplantation, for example, initial excitement was tempered by the finding of cytoplasmic transfer of reporter from donor to host cells (1). The subsequent disappointment may have discouraged investigation of whether transfer contributed to functional recovery by providing defective mutant host photoreceptors with important factors from wild-type donors. Likewise, functional recovery from bystander effects of MG reprogramming and transplantation studies may provide insights into how to preserve visual function. In short, further progress requires knowing how effects are achieved. Thus, regenerative studies in the retina must make use of developmental methods for tracking lineage and gene expression, so as to fulfill their goal of restoring vision to millions affected by blinding diseases.

\section{Acknowledgments}

We thank M. Cayouette, T. Reh, and A. Fischer for helpful discussions. This work was supported by NIH grant R01EY031685 (to SB).

Address correspondence to: Seth Blackshaw, MRB 339, 733 N. Broadway Avenue, Baltimore, Maryland 21287, USA. Phone: 443.287.5609; Email: sblack@jhmi.edu.

1. Boudreau-Pinsonneault C, Cayouette M. Cell lineage tracing in the retina: Could material transfer distort conclusions? Dev Dyn. 2018;247(1):10-17.

2. Wan J, Goldman D. Retina regeneration in zebrafish. Curr Opin Genet Dev. 2016;40:41-47.

3. Jorstad NL, et al. Stimulation of functional neuro- nal regeneration from Müller glia in adult mice. Nature. 2017;548(7665):103-107.

4. Jorstad NL, et al. STAT signaling modifies Ascl1 chromatin binding and limits neural regeneration from Muller glia in adult mouse retina. Cell Rep. 2020;30(7):2195-2208.e5.

5 . Hoang T, et al. Gene regulatory networks controlling vertebrate retinal regeneration. Science. 2020;370(6519):eabb8598.

6. Fu X, et al. Visual function restoration in genetically blind mice via endogenous cellular reprogramming [preprint]. https://doi. org/10.1101/2020.04.08.030981. Posted on bioRxiv April 8, 2020.

7. Zhou H, et al. Glia-to-neuron conversion by CRISPR-CasRx alleviates symptoms of neurological disease in mice. Cell. 2020;181(3):590-603.e16.

8. Xiao D, et al. Directed robust generation of functional retinal ganglion cells from Müller glia [preprint]. https://doi.org/10.1101/735357. Posted on bioRxiv August 14, 2019.

9. Yao K, et al. Restoration of vision after de novo genesis of rod photoreceptors in mammalian retinas. Nature. 2018;560(7719):484-488.

10. Wei X, et al. Regeneration of functional retinal ganglion cells by neuronal identity reprogramming [preprint]. https://doi. org/10.1101/2020.07.16.203497. Posted on bioRxiv July 17, 2020.

11. He Z, Jin Y. Intrinsic control of axon regeneration. Neuron. 2016;90(3):437-451.

12. Martin JF, Poché RA. Awakening the regenerative potential of the mammalian retina. Development. 2019;146(23):dev182642.

13. Wang L-L, et al. Rapid and efficient in vivo astrocyte-to-neuron conversion with regional identity and connectivity? [preprint]. https:// doi.org/10.1101/2020.08.16.253195. Posted on bioRxiv August 16, 2020.

14. Mahato B, et al. Pharmacologic fibroblast reprogramming into photoreceptors restores vision. Nature. 2020;581(7806):83-88.

15. Roth BL. DREADDs for neuroscientists. Neuron. 2016;89(4):683-694 